\title{
CONTROLE DE CAPIM-COLONIÃO NA CULTURA DA CANA-DE-AÇÚCAR COM HERBICIDAS APLICADOS EM PRÉ-EMERGÊNCIA
}

\begin{abstract}
Aimar F. F. Pedrinho Junior ${ }^{1}$ e Julio C. Durigan ${ }^{2}$
'Graduando em Agronomia. FCAV/UNESP. Jaboticabal, SP 14884-900 aimarpjr@zipmail.com.br

${ }^{2}$ Professor Titular. Departamento de Fitossanidade, FCAV/UNESP. icdurigan@fcav.unesp.br

RESUMO

Avaliou-se a eficácia dos herbicidas imazapic, imazapyr e pendimethalin, aplicados em pré-emergência na cultura da cana-de-açúcar, para controle de capim-colonião (Panicum maximum Jacq.).O trabalho foi realizado na Usina São Carlos, Jaboticabal-SP, em solo de classe textural argilosa. O delineamento experimental utilizado foi o de blocos ao acaso com quatro repetições. Os tratamentos foram: imazapic (73,5 e $98 \mathrm{~g} / \mathrm{ha})$, imazapyr (122 e $147 \mathrm{~g} / \mathrm{ha})$, imazapic+pendimethalin $(50+1000$ e $60+1200 \mathrm{~g} / \mathrm{ha})$ e pendimethalin (1200 g/ha), além das testemunhas capinada e sem capinas. Para aplicação dos herbicidas foi utilizado um pulverizador costal, à pressão constante (mantida por ar comprimido) de $2,8 \mathrm{~kg} / \mathrm{cm}^{2}, \mathrm{com} \mathrm{bicos} \mathrm{de}$ jato plano ("leque") DG 11002 e consumo de calda equivalente a 250 l/ha. A variedade de cana-de-açúcar utilizada foi a RB $72454,3^{\circ}$ corte. Tanto o imazapic como o imazapyr proporcionaram resultados considerados muito pobres no controle de $P$. maximum. A mistura de imazapic + pendimethalin apresentou excelente desempenho no controle desta espécie, podendo ser utilizada na menor dose testada. O pendimethalin isolado (1200 g/ha) foi levemente inferior às misturas, mas com resultados ainda considerados muito bons, podendo também ser recomendado para a cultura.
\end{abstract}

Palavras-chave: controle químico, imazapic, imazapyr, Panicum maximum, pendimethalin.

\section{ABSTRACT \\ Control of Panicum maximum in sugar cane with preemergence herbicides}

The major problem with the sugar cane crop is weed competition, specially Panicum maximum Jacq., which is a very agressive plant. The trial was carried out at Usina São Carlos (a sugar will), in Jaboticabal-SP, during 1998/1999 aiming to study imazapyr and imazapic herbicides, sprayed alone in preemergence, or imazapic in mixture with pendimethalin, for $P$. maximum control. The experimental design was a randomized block with nine treatments and four replications. The treatments were: controls (with and without weeds), imazapic (73.5 and $98 \mathrm{~g} / \mathrm{ha}$ ), imazapyr (122 and $147 \mathrm{~g} / \mathrm{ha})$; imazapic+pendimethalin $(50+1000$ and $60+1200 \mathrm{~g} / \mathrm{ha})$ and pendimethalin $(1200 \mathrm{~g} / \mathrm{ha})$ at preemergence. The herbicides were applied with a manual sprayer, with constant pressure of $2.8 \mathrm{~kg} / \mathrm{cm}^{2}$ and jet nozzles with descontinuous deposition (DG11002), using 250 1/ha of the herbicide solution. The sugar-cane variety used was RB 72454 . The best preemergence weed control was observed with imazapic+pendimethalim in all treatments 126 days after application. The postemergence treatment cannot be used because they caused high crop toxicity. The herbicide mixture (imazapic+pendimethalin) showed low efficiency in all tested treatments.

Key words: chemical control, imazapic, imazapyr, pendimethalin. 


\section{INTRODUÇÃO}

Um dos pontos mais críticos no processo produtivo da cana-de-açúcar é, sem dúvida, a interferência negativa imposta pelas plantas daninhas que infestam as áreas cultivadas. Essas plantas podem competir por recursos limitados do meio, hospedar pragas e doenças comuns à cultura e interferir no rendimento da colheita. Podem ainda causar reduções na quantidade e qualidade do produto colhido, além de diminuir o número de cortes economicamente viáveis (Kuva, 1999).

Dentre estas plantas, destaca-se o capim-colonião (Panicum maximum Jacq.), planta daninha comum e problemática em canaviais instalados em áreas de pastagens degradadas, formadas principalmente por capim-colonião e capimbraquiária. $\mathrm{O}$ fato de se utilizar os mesmos produtos e mesmas estratégias de controle durante muitos anos, favorece e aumenta nestas áreas a densidade de algumas espécies, entre elas, o capim-colonião. Planta de difícil controle e porte elevado, sua interferência tem causado reduções quantitativas e qualitativas na produção, principalmente nas regiões tropical e subtropical, onde adaptou-se com muita facilidade. Segundo Kuva (1999), a infestação de $P$. maximum na cultura da cana-de-açúcar pode causar redução superior a $50 \%$ na produtividade.

Devido às extensas áreas de plantio e a dificuldade de controle das plantas daninhas, o controle químico de invasoras nos canaviais é, por enquanto, a melhor alternativa para os produtores. A utilização deste método minimiza mão-deobra e tempo, constituindo, na maioria das vezes, no menos dispendioso. Atualmente, o controle do capim-colonião na maioria das usinas de açúcar e álcool é feito quimicamente pelo método da "catação", que consiste em um grupo de aplicadores procurar e aplicar isoladamente produtos de contato ou inibidores de EPSPs em cada touceira da planta. Este método acaba onerando o sistema de produção por necessitar de muita mão-de-obra, ser muito trabalhoso e consumir muito tempo.

O imazapic é um herbicida indicado e registrado para as culturas do amendoim e da cana-de-açúcar, proporcionando excelente controle de plantas daninhas e produtividades satisfatórias, mesmo causando injúrias nas fases iniciais das plantas de interesse econômico. Na cana-de-açúcar este produto ainda tem uso limitado e pouco se conhece a respeito dos sintomas de intoxicação nas plantas, sendo necessário mais pesquisas para verificar esses efeitos (Azânia, 2000).

Na cultura do amendoim, após o segundo ano de uso, Richburg et al (1995) constataram controle de 100\% para a infestação de Cyperus esculentus com o uso de imazapic (54 e $72 \mathrm{~g} / \mathrm{ha}$ ), aplicado em pós-emergência das plantas infestantes.

Shaw \& Wixson (1991), na cultura da soja, mostraram que a aplicação em pós-emergência, de imazapic $(70 \mathrm{~g} /$ ha) misturado com imazaquim (140 g/ha), causou injúrias de 8\%, 56 dias após o tratamento (DAT). A mudança na épo- ca de aplicação destes mesmos agroquímicos, injuriou mais as plantas de soja quando realizada no estádio inicial do seu desenvolvimento (Wixson \& Shaw, 1991b). Segundo estes autores, o uso de imazaquim (140 g/ha), em pré-emergência, seguido de imazapic (35g/ha), em pós-emergência inicial, proporcionou injúrias de até $13 \%$, aos 56 DAT.

Os herbicidas imazapyr e tebuthiuron também causam fitotoxicidade inicial. Imazapyr (560 g/ha) proporcionou injúrias superiores a $58 \%$ em trigo e $10 \%$ em sorgo, mesmo plantados aos 122 e 360 DAT (Schoenhals et al, 1990). Entretanto, Riley e Shaw (1998) verificaram que imazapic (105 g/ha) injuriou menos a soja, que apresentou melhor produtividade.

Em cana-de-açúcar, Richard Jr. (1997) constatou que imazapyr (1120 g/ha) não alterou a quantidade de açúcar teoricamente recuperável (ATR) e a produtividade agrícola. Porém, em outro trabalho, Richard Jr. (1998) também verificou que imazapyr (284 g/ha), aplicado por duas vezes, reduziu o estande, a altura e o ATR da cana-de-açúcar.

Akanda et al. (1997) compararam os herbicidas imazapic e imazapyr quanto ao controle de Solanum viarum e Paspalum notatum. Os autores demonstraram que imazapic (100 g/ha) proporcionou controle superior a 90\% e imazapyr $(1200 \mathrm{~g} / \mathrm{ha})$ a $100 \%$, para ambas as plantas daninhas no estágio inicial de desenvolvimento. Quando a mesma aplicação foi realizada em estádio mais desenvolvido das plantas, o controle foi de $100 \%$ para imazapyr e menor que $60 \%$ para imazapic.

Sintomas de fitointoxicação, com reflexos negativos ao desenvolvimento da cana-de-açúcar, foram observados por Blanco et al. (1983) ao aplicarem tebuthiuron $(800$ e $1200 \mathrm{~g} /$ ha) uma semana após o plantio. Coleti \& Nunes Jr. (1982) testaram tebuthiuron sobre 16 cultivares de cana-de-açúcar e verificaram que alguns cultivares são mais susceptíveis que outros. O dobro da dose recomendada de thebuthiuron causou menores sintomas iniciais de fitointoxicação para os cultivares IAC 48-65, IAC 52-150, NA 56-79, SP 70-1143 e SP 71-3149, enquanto na dose recomendada os cultivares mais susceptíveis foram IAC 51-205, CB 45-155, CP 51-22 e SP 70-1078. No entanto, Graciano \& Ramalho (1982) e Rolim \& Christoffoleti (1982) não demonstraram alterações de produtividade e qualidade agrícola nos diferentes cultivares submetidos à aplicação de tebuthiuron $(800,1200,1600,1800 \mathrm{e}$ $2400 \mathrm{~g} / \mathrm{ha})$.

Durigan (1993) relata que a mistura de produtos pode trazer efeitos sinérgicos, aditivos ou antagônicos à ação dos herbicidas, dependendo do grupo químico e/ou classe dos mesmos. A eficiência destas, também é muito influenciada pelos tipos de plantas daninhas que se visa controlar e a cultura em que estão ocorrendo. $\mathrm{O}$ uso de surfactantes e aditivos associados à imazapic em pós-emergência, colaborou com o aumento da toxicidade às plantas da soja (Wixson \& Shaw, 1991a).

Para minimizar os efeitos da fitointoxicação do herbicida pode-se associá-lo ao cultivo, pois conforme Newson 
\& Shaw (1994), doses menores de imazapic (9 g/ha), em pósemergência, seguidas de cultivo, aos sete dias após o tratamento, proporcionam menores injúrias.

Com o objetivo de estabelecer novas alternativas para o controle químico do capim-colonião na cultura da cana de açúcar, conduziu-se um experimento com os herbicidas imazapic, isolado e em mistura com pendimethalin, e imazapyr e pendimethalin isolados, aplicados em pré-emergência da cultura e das plantas daninhas, procurando também verificar a seletividade para as plantas cultivadas.

\section{MATERIAL E MÉTODOS}

O experimento foi instalado em área da Usina São Carlos, no município de Jaboticabal-SP, no ano agrícola de 1998/99. O clima da região, segundo a classificação de Köeppen, é do tipo Cwa (mesotérmico de inverno seco), com temperatura média no mês mais quente (janeiro) de $22^{\circ} \mathrm{C}$ e no mês mais frio (julho) de $18^{\circ} \mathrm{C}$.

A análise granulométrica do solo da área experimental apresentou os seguintes resultados (em g/kg): argila 360, limo 430, areia fina 170 e areia grossa 40, sendo classificado na classe textural argilosa.

O delineamento experimental utilizado foi o de blocos ao acaso, com nove tratamentos e quatro repetições. As parcelas experimentais constaram de três linhas espaçadas de $1,4 \mathrm{~m}$ e com $8 \mathrm{~m}$ de comprimento, perfazendo $33,6 \mathrm{~m}^{2}$. Os herbicidas testados, com as respectivas doses, são apresentados na Tabela 1 .

O herbicida imazapic pertence ao grupo químico das imidazolinonas e apresenta-se na formulação de grânulos dispersíveis em água (GRDA), contendo $700 \mathrm{~g} / \mathrm{kg}$ do ingrediente ativo e classe toxicológica III, faixa azul, medianamente tóxico. O imazapyr também pertencente ao grupo químico das imidazolinonas, apresenta-se na formulação solução aquosa concentrada ( SaqC) e classe toxicológica III. O pendimethalin, utilizado na formulação concentrado emulsionável (CE), contém $500 \mathrm{~g} / \mathrm{l}$ de ingrediente ativo, pertence à classe toxicológica II (Rodrigues \& Almeida, 1998).

Para a aplicação utilizou-se de um pulverizador costal, à pressão constante (mantida por ar comprimido) de $2,8 \mathrm{~kg} /$ $\mathrm{cm}^{2}$, munido de barra com quatro bicos de jato plano (tipo "leque") DG 11002 e consumo de calda equivalente a $250 \mathrm{l}$ ha.

Na aplicação em pré-emergência, dia 30/11/98, o solo encontrava-se com excelente umidade, temperatura ambiente de $36^{\circ} \mathrm{C}$, temperatura do solo (a $5 \mathrm{~cm}$ de profundidade) de $38,0^{\circ} \mathrm{C}$, nebulosidade em torno de $50 \%$, ventos intermitentes (2 a $4 \mathrm{~km} / \mathrm{h}$ ) e umidade relativa do ar de $53 \%$. A variedade de cana-de-açúcar utilizada no experimento foi a RB72454, no $3^{\prime \prime}$ corte.

As avaliações de controle das plantas daninhas foram realizadas aos 30, 70, 91 e 126 dias após a aplicação (DAA), atribuindo-se visualmente as percentagens de $100 \%$ para controle total, e $0 \%$ para a ausência dele. Para o capim-colonião, pode-se considerar controle aceitável com percentagens acima de $90 \%$. As avaliações dos sintomas de fitointoxicação na cultura, foram realizadas segundo o método proposto pela SBCPD (1995), onde as letras de A a E correspondem aos danos causados às plantas, sendo $\mathrm{A}=$ nenhum dano $\mathrm{e} \mathrm{E}=$ morte da planta. Foram feitas quatro avaliações de fitotoxicidade, segundo esse método, todas nas mesmas datas das avaliações de controle. Aos $70 \mathrm{DAA}$ foi efetuada a medição de altura das plantas de cana-de-açúcar até a última aurícula visível.

Os dados foram submetidos à análise de variância estatística e submetidos ao teste F. Para a diferenciação das médias utilizou-se o teste de Tukey, a $5 \%$ de probabilidade. Os dados originais foram transformados em arco sen $\sqrt{\mathrm{x}}$ para possibilitar melhor normalização dos dados e melhor adequação para as análises estatísticas.

\section{RESULTADOS E DISCUSSÃO}

Os dados diários de temperatura média, precipitação e umidade relativa do ar, ocorridos durante o experimento, são apresentados na Figura 1. Pode-se observar que, no decorrer do experimento, os parâmetros metereológicos avaliados sempre estiveram acima dos níveis mínimos necessários. A temperatura média sempre esteve acima de $20^{\circ} \mathrm{C}$, possibi-

Tabela 1. Herbicidas e doses, aplicados em pré-emergência na cultura da cana-de-açúcar. Jaboticabal, SP. 1998/1999.

\begin{tabular}{lcccc}
\hline \multicolumn{2}{c}{ Herbicida } & & \multicolumn{2}{c}{ Doses } \\
\cline { 1 - 2 } \multicolumn{1}{c}{ Nome comum } & Nome comercial & & $\begin{array}{c}\text { Ingrediente ativo } \\
\text { (g/ha) }\end{array}$ & $\begin{array}{c}\text { Produto comercial } \\
\text { (l ou g/ha) }\end{array}$ \\
\hline Imazapic & Plateau 70 DG & Plateau 70 DG & 73,5 & $105 \mathrm{~g}$ \\
Imazapic & Contain & Contain & 98 & $140 \mathrm{~g}$ \\
Imazapyr & Plateau+Herbadox & & $50+1000$ & 0,51 \\
Imazapyr & Plateau+Herbadox & & $60+1200$ & $71 \mathrm{~g}+2,01$ \\
Imazapic+pendimethalin & Herbadox 500 CE & & 1200 & $85 \mathrm{~g}+2,41$ \\
Imazapic+pendimethalin & Pendimethalin & & & 2,41 \\
\hline
\end{tabular}




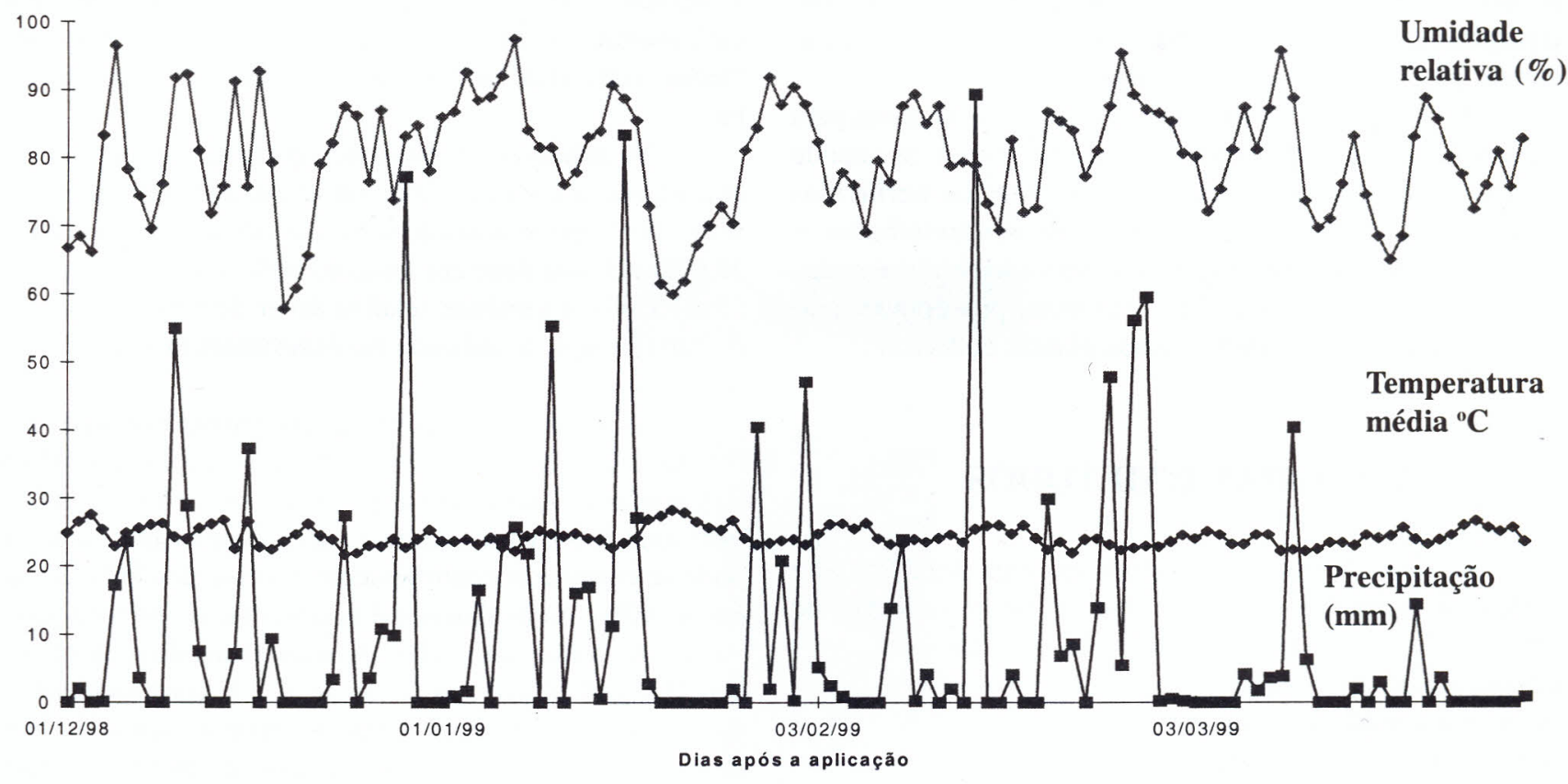

Figura 1. Temperatura média, precipitação diária e umidade relativa do ar; durante os meses de dezembro de 1998 a março de 1999. Jaboticabal, SP. 1998/99. (Fonte: FCAV/UNESP).

litando uma taxa metabólica elevada das plantas. A umidade relativa do ar nunca esteve abaixo de $50 \%$, propiciando boas condições para a absorção dos produtos. Houve uma precipitação relativamente alta durante a condução do experimento, porém, não atingindo níveis que pudessem prejudicar a ação do herbicida no solo.

A principal planta daninha de ocorrência na área experimental era capim-colonião (Panicum maximum Jacq.), cobrindo, em média, $75 \%$ do solo no momento da aplicação. Os resultados de controle apresentados na Tabela 2, mostram aos $30 \mathrm{DAA}$, que imazapic isolado não proporcionou índices satisfatórios de controle, bem como a menor dose de imazapyr (122 g/ha). Alves et al. (1997) obtiveram excelente desempenho destes dois herbicidas, até 120 dias após a aplicação, para o controle de capim-colchão (Digitaria horizontalis) na cultura da cana-de-açúcar, quando aplicados 40 dias antes do plantio da cultura. Os tratamentos com a mistura de imazapic+pendimethalin $(50+1000$ e $60+1200 \mathrm{~g} / \mathrm{ha})$ nas duas doses testadas, bem como pendimethalin isolado (1200 g/ ha), proporcionaram excelente controle e diferiram estatisticamente dos demais. Resultados diferentes foram relatados por Paula Neto et al. (2000a), que observaram uma atuação pior da mesma mistura, nas mesmas doses, para o controle de capim-braquiária (Brachiaria horizontalis) na cultura da cana-de-açúcar.

Aos 70 DAA, a maior dose de imazapyr (147 g/ha) não apresentava bom controle. Essa tendência foi mantida até a última avaliação, aos 126 DAA, com excelente controle proporcionado pelas misturas bem como pelo pendimethalin isolado. Em outro trabalho, Paula Neto et al. (2000b) relatam ótimo controle, em pré-emergência, da comunidade infestante da cultura da cana-de-açúcar, proporcionada tanto por imazapic isolado quanto em mistura com pendimethalin, ambos nas mesmas doses testadas no presente estudo. Os resultados indicaram com clareza a importância do pendimethalin, em mistura com imazapic, para controle do capim-colonião. Constatou-se boa atividade do pendimethalin mesmo quando aplicado isoladamente, proporcionando resultados excelentes de controle.

Com relação aos conceitos atribuídos nas avaliações visuais para caracterização ou não da seletividade (Tabela $3)$, verificou-se leve redução de altura em todos os tratamentos aplicados. Porém, na avaliação realizada aos 70 DAA, os dados analisados estatisticamente já não diferiram entre si, demonstrando a recuperação das plantas de cana-de-açúcar. A fitotoxicidade visualisada nos primeiros 70 dias, caracterizada com conceito B (injúrias leves), também foi observada por Alves et al. (1997), Paula Neto et al. (2000a) e Paula Neto et al. (2000b), com os mesmos herbicidas e misturas.

Pedrinho Jr. et al. (2001), observaram que o herbicida imazapic aplicado em pré-emergência, nas doses de 122,5 e 147,0 g/ha, causou fitotoxicidade (nível C) às plantas de canade-açúcar da variedade RB 855536, até noventa dias após a aplicação. Os mesmos relataram que o herbicida imazapic aplicado em pós-emergência na cultura da cana-de-açúcar, para a variedade citada, proporcionou fortes sintomas de intoxicação nas plantas, recebendo até conceito D, inviabilizando o seu uso.

Segundo Wixson \& Shaw (1992), a aplicação de imazapic $(9,18$ e $35 \mathrm{~g} / \mathrm{ha})$, em pré-emergência, também causou até $20 \%$ de injúria inicial às plantas de diferentes culturas, como a soja, o algodão e o milho. 
Tabela 2. Percentagens de controle de capim-colonião (Panicum maximum), em diferentes épocas após a aplicação dos herbicidas em pré emergência, na cultura da cana-de-açúcar. Jaboticabal, SP. 1998/1999.

\begin{tabular}{|c|c|c|c|c|c|c|}
\hline \multicolumn{2}{|l|}{ Tratamento } & \multicolumn{5}{|c|}{ Época de avaliação (DAA) } \\
\hline Nome comum & Dose (g/ha) & 30 & 70 & \multicolumn{2}{|c|}{91} & 126 \\
\hline Imazapic & 73,5 & ${ }^{\mathrm{a}} 61 \mathrm{c} \quad(74)^{\mathrm{b}}$ & $46 \mathrm{c} \quad(52)$ & $36 \mathrm{de}$ & $(35)$ & $34 \mathrm{~cd} \quad(32)$ \\
\hline Imazapic & 98 & $71 \mathrm{~b} \quad(89)$ & $61 \mathrm{~b}$ & $49 \mathrm{~cd}$ & (58) & $46 \mathrm{bc} \quad(51)$ \\
\hline Imazapyr & 122 & $69 \mathrm{bc} \quad(86)$ & $44 \mathrm{c} \quad(48)$ & $30 \mathrm{e}$ & (26) & $30 \mathrm{~d} \quad(25)$ \\
\hline Imazapyr & 147 & $74 \mathrm{~b} \quad(92)$ & 52 bc (62) & $51 \mathrm{c}$ & $(60)$ & $49 \mathrm{~b} \quad(58)$ \\
\hline Imazapic+pendimethalin & $50+1000$ & 90 a (100) & 90 a $(100)$ & $86 a b$ & (99) & $90 \mathrm{a} \quad(100)$ \\
\hline Imazapic+pendimethalin & $60+1200$ & 90 a (100) & 90 a $(100)$ & $85 \mathrm{ab}$ & $(98)$ & 85 a (98) \\
\hline Pendimethalin & 1200 & 90 a (100) & 87 a (98) & $76 \mathrm{~b}$ & (94) & 79 a \\
\hline Testemunha sem capina & - & $0 \mathrm{~d} \quad(0)$ & $0 \mathrm{~d} \quad(0)$ & $0 \mathrm{f}$ & (0) & $0 \mathrm{e} \quad(0)$ \\
\hline Testemunha capinada & - & 90 a (100) & 90 a (100) & $90 \mathrm{a}$ & $(100)$ & 90 a $\quad(100)$ \\
\hline $\mathrm{F}$ & & $301,24 * *$ & $145,31 * *$ & 113, & $74 * *$ & $136,85 * *$ \\
\hline DMS & & 7,95 & 12,02 & 13 & & 13,08 \\
\hline C.V. (\%) & & 4,7 & 8,1 & 10, & & 9,7 \\
\hline
\end{tabular}

${ }^{a}$ Dados transformdos em arco sen $\sqrt{\mathrm{x}}$

${ }^{\mathrm{b}}$ Dados originais

DAA - dias após a aplicação

Tabela 3. Conceitos atribuídos aos sintomas visuais de intoxicação na cultura da cana-de-açúcar e altura de plantas aos 70 dias após a aplicação (DAA). Jaboticabal, SP. 1998/1999.

\begin{tabular}{|c|c|c|c|c|c|c|}
\hline \multirow{2}{*}{ Tratamento } & \multirow{2}{*}{$\begin{array}{c}\text { Dose } \\
\text { (g/ha) }\end{array}$} & \multicolumn{4}{|c|}{ Época de avaliação (DAA) } & \multirow{2}{*}{$\begin{array}{c}\text { Altura } \\
\text { média } \\
(\mathbf{c m})\end{array}$} \\
\hline & & 30 & 70 & 91 & 126 & \\
\hline Imazapic & 73,5 & B & $\mathrm{B}$ & A & A & 49,92 \\
\hline Imazapic & 98 & B & $\mathrm{B}$ & A & A & 44,20 \\
\hline Imazapyr & 122 & B & B & A & A & 48,92 \\
\hline Imazapyr & 147 & B & $\mathrm{B}$ & A & A & 46,72 \\
\hline Imazapic+pendimethalin & $50+1000$ & $\mathrm{~B}$ & $\mathrm{~B}$ & A & A & 41,97 \\
\hline Imazapic+pendimethalin & $60+1200$ & $\mathrm{~B}$ & B & A & A & 50,50 \\
\hline Pendimethalin & 1200 & A & A & A & A & 50,15 \\
\hline Testemunha sem capina & - & A & A & A & A & 51,20 \\
\hline Testemunha capinada & - & A & A & A & A & 47,85 \\
\hline $\mathrm{F}$ & & & & & & $0,67^{\mathrm{ns}}$ \\
\hline C.V. (\%) & & & & & & 15,9 \\
\hline DMS & & & & & & 18,37 \\
\hline
\end{tabular}

O resultado obtido por Azânia (2000) mostra que, para a dose de $122,5 \mathrm{~g} / \mathrm{ha}$ de imazapic, a fitotoxicidade foi pequena, com notas iguais ou inferiores a 2 , segundo a escala da EWRC (1964), aos 30 DAA.

\section{CONCLUSÕES}

O imazapic (73,5 e $98 \mathrm{~g} / \mathrm{ha})$ e o imazapyr (122 e 147 $\mathrm{g} / \mathrm{ha}$ ) proporcionaram resultados considerados como muito baixos para o controle de Panicum maximum.
A mistura imazapic+pendimethalin $(50+1000$ e $60+$ $1200 \mathrm{~g} / \mathrm{ha}$ ) evidenciou excelente desempenho, podendo-se utilizar a menor dose para o controle desta espécie.

Pendimethalin isolado (1200 g/ha) foi levemente inferior às suas misturas com imazapic mas também com resultados considerados ótimos.

Com exceção do pendimethalin aplicado isoladamente, todos os demais herbicidas e misturas estudadas, causaram leves injúrias às plantas de cana-de-açúcar, que desapareceram completamente com o transcorrer do tempo. 


\section{LITERATURA CITADA}

AKANDA, R.V.; MULLAHEY, J.J.; DOWLER, C.C.; SHILLING, D.G. Influence of postemergence herbicides on tropical soda apple (Solanum viarum) and bahiagrass (Paspalum notatum). Weed Technology, v.11, p. 656$661,1997$.

ALVES, P.L.C.A.; KAWAGUCHI, I.T.; PICCIN, C.H.; LUSVARGHI, H. Comportamento dos herbicidas imazapyr e imazameth sobre uma comunidade de plantas daninhas infestando a cultura da cana-de-açúcar. In: CONGRESSO BRASILEIRO DA CIÊNCIA DAS PLANTAS DANINHAS, 21. Caxambú-MG, 1997. Resumos... Belo Horizonte: SBCPD, 1997. p. 239.

AZÂNIA, C. A. M. Controle de plantas infestantes com diferentes herbicidas e sua seletividade às soqueiras de cana-de-açúcar (Saccharum spp.). Faculdade de Ciências Agrárias e Veterinárias -UNESP, Campus de Jaboticabal. Jaboticabal-SP, 2000. (Dissertação de Mestrado).

BLANCO, H.G.; OLIVEIRA, D.A.; COLETI, J.T.; TERAO, D. Fitotoxicidade em cana-de-açúcar (Saccharum spp), em solo arenoso, induzida pela interação de tebuthiuron e carbofuran. Biológico, v. 49, p.227-236, 1983.

COLETI, J.T.; NUNES JR, D. Avaliação inicial da fitotoxicidade de herbicidas sobre 16 cultivares de canade-açúcar (Saccharum spp). In: CONGRESSO BRASILEIRO DE HERBICIDAS E ERVAS DANINHAS, 14 e CONGRESO DE LA ASOCIACION LATINO AMERICANA DE MALEZAS, 6. Campinas, 1982. Resumos... Campinas: SBHED, 1982. p.89.

DURIGAN, J.C. Efeitos de adjuvantes na aplicação e eficácia dos herbicidas. FUNEP, 1993. 42p. (Boletim Científico).

EUROPEAN WEED RESEARCH COUNCIL (EWRC). Report of the $3^{\text {rd }}$ and $4^{\text {rd }}$ meetings of EWRC: Committee of Methods in Weed Research. Weed Research, v.4, p.88, 1964.

GRACIANO, P.C.; RAMALHO, J.F.G.P. Susceptibilidade do cultivar CB 45-03 de cana-de-açúcar (Saccharum spp.) ao herbicida thebuthiuron. In: CONGRESSO BRASILEIRO DE HERBICIDAS E ERVAS DANINHAS, 14 e CONGRESO DE LA ASOCIACION LATINO AMERICANA DE MALEZAS, 6. Campinas, 1982. Resumos... Campinas: SBHED, 1982. p.91.

KUVA, M.A. Efeito de período de controle e de convivência das plantas daninhas na cultura da cana-de-açú- car (Saccharum sp) no Estado de São Paulo. Escola Superior de Agricultura Luiz de Queiroz. PiracicabaSP. 1999. 74p. (Dissertação de Mestrado)

NEWSON, L.J.; SHAW, D.R. Influence of cultivation timing on weed control in soybean (Glycine max) with AC 263,222. Weed Tecnology., v.8, p. 760-765, 1994.

PAULA NETO, J.F.; ALVES, P.L.C.A.; BACHEGA, M.F.; SOUZA, J.C. Avaliação do imazapic, aplicado isolado ou em mistura com pendimethalin, e do imazapyr no controle de uma população de capim-braquiária e de sua seletividade para a cultura da cana-de-açúcar. In: CONGRESSO BRASILEIRO DA CIÊNCIA DAS PLANTAS DANINHAS, 21. Foz do Iguaçu-PR, 2000. Resumos... Foz do Iguaçu: SBCPD, 2000a. p. 306.

PAULA NETO, J.F.; ALVES, P.L.C.A.; MATTOS, E.D; BACHEGA, M.F.; MARCON, V.M. Avaliação da eficácia do imazapic no controle de uma comunidade de plantas daninhas e sua seletividade para a cultura da cana-de-açúcar. In: CONGRESSO BRASILEIRO DA CIÊNCIA DAS PLANTAS DANINHAS, 21. Foz do Iguaçu-PR, 2000. Resumos... Foz do iguaçu: SBCPD, 2000b. p. 307.

PEDRINHO JR., A.F.F.; MARTINI, G.; DURIGAN, J.C. Controle de tiririca (Cyperus rotundus) na cultura da cana-de-açúcar, com o herbicida imazapic isolado ou em mistura com pendimethalin. Revista Brasileira de Herbicidas, v.2, n.1, p. 23-30, 2001.

RICHARD JR., E.P. Effects of fallow bermudagrass (Cynodon dactylon) control programs on newly planted sugarcane (Saccharum spp. hybrids). Weed Technology, v.11, p.677-682, 1997.

RICHARD JR., E.P. Control of perennated bermudagrass (Cynodon dactylon) and johnsongrass (Sorghum halepense) in sugarcane (Saccharum spp. hybrids). Weed Technology, v.12, p.128-133, 1998.

RICHBURG, J.S.; WILCUT, J.W.; WILLEY, G.L. AC 263,222 and imazethapyr rates and mixture for weed management in peanut (Arachis hypogaea). Weed Technology, v.9, p.801-806, 1995.

RILEY, D.G., SHAW, D.R. Influence of imazapyr on the con trol of pitted morningglory (Ipomoea lacunosa) and johnsongrass (Sorghum halepense) with chlorimuron, imazaquin, and imazethapyr. Weed Science, v.36, p.663-666, 1998.

RODRIGUES, B.N.; ALMEIDA, F.S. Guia de herbicidas. 4 ed. Londrina: Editora do Autor, 1998. 648 p. 
ROLIM, J.C.; CHRISTOFFOLETI, P.J. Tolerância de cultivares de cana-de-açúcar (Saccharum spp) ao herbicida tebuthiuron. In: CONGRESSO BRASILEIRO DE HERBICIDAS E ERVAS DANINHAS, $14 \mathrm{e}$ CONGRESO DE LA ASOCIACION LATINO AMERICÁNA DE MALEZAS, 6. Campinas, 1982. Resumos... Campinas: SBCPD, 1982. p.90-91.

SCHOENHALS, M.G.; WIESE, A.F.; WOOD, M.L. Field bindweed (Convolvulus arvensis) control with imazapyr. Weed Technology, v.4, p.771-775, 1990.

SHAW, D.R.; WIXSON, M.B. Postemergence combinations of imazaquim or imazethapyr with AC 263,222 for weed control in soybean (Glycine max). Weed Science, v.39, p.644 - 649, 1991.
SOCIEDADE BRASILEIRA DA CIÊNCIA DAS PLANTAS DANINHAS (SBCPD). Procedimentos para instalação, avaliação e análise de experimentos com herbicidas. Londrina: SBCPD, 1995. 42p.

WIXSON, M.B.; SHAW, D.R. Effects of adjuvants on weed control and soybean (Glycine max) tolerance with AC 263,222. Weed Technology, v.5, p.817-822, 1991 a.

WIXSON, M.B.; SHAW, D.R. Use of AC 263,222 for sicklepod (Cassia obtusifolia) control in soybean (Glycine max). Weed Technology, v.5, p.434-438, $1991 \mathrm{~b}$.

WIXSON, M.B.; SHAW, D.R. Effects of soil-applied AC 263,222 on crops rotated with soybean (Glycine max). Weed Technology, v.6, p.276-279, 1992. 
